\title{
Métodos ativos de aprendizagem: o uso do aprendizado baseado em problemas no ensino de logística e transportes
}

\author{
Igor de Moraes Cardoso ${ }^{1}$ e Renato da Silva Lima ${ }^{2}$
}

\begin{abstract}
Resumo: Diversas formas de ensino têm sido avaliadas por pesquisadores preocupados em obter mais dos alunos. O aprendizado baseado em problemas (ABP ou PBL - do inglês problem-based learning) é uma dessas abordagens, e que pode ser descrito como o método de ensino-aprendizagem em que os alunos aprendem através da resolução de problemas complexos que não têm uma única resposta correta. O objetivo deste trabalho é analisar a efetividade do ABP no ensino de Logística e Transportes. Os resultados mostraram a efetividade do método através das observações realizadas e confirmaram a hipótese do modelo proposto. Os alunos consideraram este método mais dinâmico e motivador que aulas expositivas, porém reconheceram que demanda mais tempo. O docente considerou a aula mais dinâmica e desafiadora para todos, mas também mais imprevisível, pela possibilidade do levantamento de questões conceituais desconhecidas para o docente. DOI:10.4237/transportes.v20i3.561.
\end{abstract}

Palavras-chave: Aprendizado Baseado em Problemas (PBL), ensino de engenharia, logística e transportes.

\begin{abstract}
Several forms of education have been evaluated by researchers concerned in obtain more from students. The problem-based learning (or PBL) is one of these approaches, which can be described as the teaching-learning method in which students learn by solving complex problems that do not have a single correct answer. The objective of this study is to analyze the effectiveness of PBL in the teaching of Logistics and Transport. The results shown the effectiveness of the method through the observations and confirmed the hypothesis of the model. The students found this method more dynamic and motivating than lectures, but acknowledged that it demands more time. The instructor considered the class more dynamic and challenging for everyone, but also more unpredictable, due the possibility of unknown conceptual issues rising to the teacher.
\end{abstract}

Keywords: Problem-Based Learning (PBL), engineering education, logistics \& transportation.

\section{INTRODUÇÃO}

Nas atividades de ensino, o conhecimento é apresentado como primordial e, na maioria das vezes, acontece através de palestras, apresentações, testes, leituras e redações. A ação é, de forma geral, considerada indesejada na sala de aula, com estudantes em silêncio em suas carteiras, ao invés de interagindo e conversando (Crookall e Thorngate, 2009). Diversas formas de ensino têm sido avaliadas por pesquisadores preocupados em obter mais dos alunos (conhecimento, habilidades e atitudes) e comparadas à abordagem de ensino com o método tradicional. Uma dessas abordagens diz respeito ao uso de mais atividades práticas em sala de aula, com o objetivo de que o aluno seja capaz de associar os conhecimentos e habilidades adquiridos à sua vida profissional ou a uma situação particular. Essa abordagem mais prática é encontrada em diversas teorias de aprendizado: está associada ao uso de estudos de caso, à resolução de problemas baseados em projetos, ao uso de jogos e simulações etc.

Ambientes tradicionais de ensino e aprendizado são, de forma geral, previsíveis, estáticos, sem desafios e entediantes, em particular quando comparados com a televisão, internet, redes sociais e outros ambientes do mundo real. Algumas limitações do modelo tradicional de ensino são listadas por Ruben (1999):

\footnotetext{
${ }^{1}$ Igor de Moraes Cardoso, Instituto de Engenharia de Produção e Gestão, Universidade Federal de Itajubá, Itajubá, MG, Brasil. (e-mail: cardoim@gmail.com).

${ }^{2}$ Renato da Silva Lima, Instituto de Engenharia de Produção e Gestão, Universidade Federal de Itajubá, Itajubá, MG, Brasil. (e-mail: rslima@unifei.edu.br).
}

Manuscrito recebido em 8/3/2012 e aprovado para publicação em 25/9/2012 Este artigo é parte de TRANSPORTES v. 20, n. 3, 2012. ISSN: 2237-1346 (online). DOI:10.4237/transportes.v20i3.561.
- Enquanto o tradicional método de ensino e aprendizado enfatiza a transmissão do conhecimento de um reconhecido conhecedor do assunto (professor) para indivíduos (alunos), o ensino e aprendizado fora da sala de aula é predominantemente baseado em pares, mais social e colaborativo;

- Aprender o que o professor tem a intenção de ensinar não é a única consequência do ambiente ensinoaprendizado. Acomodar resultados e aplicações do aprendizado e promover a criatividade são fundamentais;

- A estrutura de classes tradicionais e as abordagens clássicas para avaliação obtêm pouco resultado ao tentar promover o aprendizado ativo; e

- O modelo tradicional de transferência de conhecimento geralmente não trata da inseparabilidade dos domínios comportamentais, cognitivos e afetivos.

Desde a década de 1960, a investigação sobre a aprendizagem das ciências tem contribuído para obtenção de melhores práticas educativas, na medida em que as abordagens pedagógicas e processos estão em pauta (Yeo, 2005). Ainda segundo Yeo (2005), tendências modernas de educação demandam que os alunos desempenhem um papel ativo no processo de obtenção de conhecimento. Um forte senso de participação é requerido de cada aluno para experimentar uma variedade de processos, que vão do autoaprendizado direto a grupos de discussão, ao ensino em pares e a orientação por professores. É nesta experiência orientada à ação que o indivíduo aprende mais. O professor, que antes era o possuidor de todo conhecimento, agora orienta, guia e habilita o aluno a tornar-se responsável por seu processo de aprendizado. $\mathrm{O}$ aprendizado geralmente é motivado por problemas da vida real, em que questões pertinentes são identi- 
ficadas e soluções em potencial discutidas e exploradas (Yeo, 2005).

$\mathrm{O}$ aprendizado independente e em time são duas características chave do aprendizado baseado em problemas (neste trabalho será usada a sigla $\mathrm{ABP}$, proveniente do inglês $\mathrm{PBL}$ - Problem-Based Learning) como componentes essenciais do processo de aprendizado (Yeo, 2005). Essa abordagem requer que o aluno adote uma mudança de mentalidade, de dependente do professor para a autossuficiência. Entretanto, muitas pessoas não estão confortáveis com esta abordagem por duas razões: professores não podem abandonar seu papel de fonte de conhecimento e os alunos estão habituados com seu papel de receptores. Para manter um equilíbrio adequado, as instituições devem estar atentas a mudanças na programação de ensino, tamanho das turmas, a definição e formatos de aula.

Nesse contexto, o objetivo principal do trabalho é analisar a efetividade do método de aprendizado baseado em problemas no ensino de Logística e Transportes. O método / problema deverá fundamentar-se na aplicação, desenvolvimento e difusão de conhecimentos e raciocínios ligados a esse tema, bem como seu uso prático. Os principais temas de Logística que devem ser abordados são: Gerenciamento da Cadeia de Suprimentos, Gestão do Relacionamento com Clientes e com Fornecedores, Canais de Distribuição e Distribuição Física, Fundamentos de Transportes, Intermodalidade e Multimodalidade, pois são os temas principais da ementa da disciplina aplicada na universidade onde o trabalho foi realizado.

Buscou-se ainda, como objetivos secundários, analisar se os alunos: obtêm uma compreensão dos problemas associados ao tema de Logística e Transportes; obtêm conhecimento da teoria aplicável deste tema; desenvolvem habilidades de resolução de problemas; desenvolvem a capacidade de pensar analiticamente; desenvolvem habilidades de apresentação oral e escrita; sabem como e onde encontrar conhecimento relevante para um dado problema; aprendem, a partir de um conjunto de fatos e situações, como definir os problemas; aprendem como aplicar o conhecimento; desenvolvem habilidades para reconhecer qual conhecimento é relevante; desenvolvem habilidades em usar tecnologia de informação (editor de textos, planilhas, etc.) e desenvolvem habilidades para aprender a trabalhar com outras pessoas em equipes.

O trabalho está estruturado da seguinte forma: após esta introdução apresenta-se, na seção 2, o referencial teórico sobre Aprendizado Baseado em Problemas. Na seção 3 apresenta-se a metodologia de pesquisa, seguida dos resultados, discutidos do ponto de vista qualitativo e quantitativo na seção 4. A seção 5 traz as conclusões do trabalho, seguidas da lista com as referências bibliográficas.

\section{APRENDIZADO BASEADO EM PROBLEMAS}

ABP é uma abordagem educativa centrada no aluno, que os habilita a realizar pesquisas, integrar a teoria e a prática, e aplicar os conhecimentos e habilidades para desenvolver uma solução viável para um problema definido. Fundamental para o sucesso da abordagem é a seleção de problemas mal estruturados (e muitas vezes interdisciplinares) e um tutor que oriente o processo de aprendizagem e realize um balanço na conclusão da experiência de aprendizagem. Savery (2006) menciona uma lista de práticas consideradas características da filosofia, estratégias e táticas de ABP, além de métodos utilizados e as competências específicas desenvolvidas. Estas incluem a capacidade de pensar criticamente, analisar e resolver os problemas complexos do mundo real, para localizar, avaliar e usar de forma adequada os recursos de aprendizagem, para trabalhar cooperativamente, para demonstrar habilidades de comunicação eficaz, e de utilizar o conhecimento e habilidades intelectuais. Segundo Kuri et al. (2007), a grande diferença entre a abordagem clássica e o ABP é que o segundo procura garantir aos alunos a compreensão dos fatos, em vez da simples memorização de conceitos. Para o professor, a abordagem também traz inúmeras vantagens, já que muitas vezes ele próprio aprende ao longo do curso, ainda que o processo seja um grande e permanente desafio (Silva et al., 2012).

Hmelo-Silver (2004) descreve o ABP como o método de ensino em que os alunos aprendem através da resolução de problemas e que foca em um problema complexo que não tem uma única resposta correta. Ela observou que os alunos trabalham em grupos colaborativos para identificar o que precisam aprender para resolver um problema, se envolver na autoaprendizagem, aplicar seus novos conhecimentos para o problema, e refletir sobre o que aprenderam e a eficácia das estratégias empregadas. Enquanto nos métodos "tradicionais" de ensino primeiro são colocados os conceitos e depois os problemas ou exercícios de aplicação, no PBL acontece o contrário: as aulas se iniciam com a proposição de um problema, engajando os alunos com o conteúdo da disciplina via atividade e descoberta (Kuri e Silva, 2010; Silva et al., 2012).

\subsection{Características do ABP}

Algumas das características essenciais do ABP são apresentadas a seguir (Savery, 2006):

- A responsabilidade pelo próprio aprendizado deve ser do estudante;

- O ABP é uma abordagem focada no aluno. Os estudantes se empenham no problema com o que seu conhecimento e experiência atual permitem. A motivação aumenta quando a responsabilidade para a solução do problema e do processo é do aluno;

- Os problemas simulados em ABP devem ser mal estruturados e permitir o livre questionamento;

- Os problemas no mundo real são mal estruturados. Uma habilidade desenvolvida através do ABP é a de identificar problemas e determinar parâmetros para o desenvolvimento da solução. Quando um problema é bem estruturado, os alunos são menos motivados e investem menos no desenvolvimento da solução;

- O aprendizado deve ser integrado através de uma grande variedade de temas e disciplinas;

- Durante o aprendizado autodirigido, os estudantes devem ser capazes de acessar, estudar e integrar informação de diversas disciplinas que possam estar relacionadas ao entendimento e resolução de um problema em particular;

- A colaboração é essencial;

- No mundo além da escola ou da universidade, a maioria dos alunos irá se deparar com atividades profissionais em que precisam compartilhar informações e trabalhar de forma mais produtiva com outras pessoas. O ABP fornece o formato para o desenvolvimento dessas habilidades; 
- O ponto de pesquisa autodirigida é para os indivíduos coletarem informações que serão levadas ao grupo para o processo de tomada de decisão em relação ao problema. É essencial que cada indivíduo compartilhe coerentemente o que aprendeu e como esta informação pode impactar no desenvolvimento da solução para o problema;

- Uma análise de fechamento do que foi aprendido com o problema e uma discussão de quais conceitos foram aprendidos é essencial;

- Dado que o ABP é uma forma de aprendizado experimental, os alunos geralmente ficam muito próximos dos detalhes do problema e da solução proposta. O propósito do processo de discussão após a experiência é consolidar o aprendizado;

- Processos de autoavaliação e avaliação por pares devem ser conduzidos ao término de cada problema e ao final de cada unidade curricular. Essas avaliações são relacionadas à característica prévia de reflexão sobre o ganho de conhecimento. O significado desta atividade é reforçar a natureza de autorreflexão do aprendizado;

- As atividades realizadas em uma ABP devem ser aquelas valorizadas no mundo real;

- ABP deve ser a base de um currículo pedagógico e não parte de um currículo didático;

- As avaliações dos estudantes devem medir o progresso através dos objetivos do ABP; e

- Os objetivos do ABP são baseados em conhecimento e processo. Os estudantes precisam ser avaliados nestas dimensões, em intervalos regulares, para garantir o benefício que se espera da abordagem do ABP. Eles precisam ser capazes de reconhecer e enunciar o que sabem e o que aprenderam.

Essas descrições das características essenciais claramente identificam:

- O papel do tutor como um facilitador do aprendizado;

- As responsabilidades dos aprendizes de serem autodirecionados e autorregulados; e

- Que os elementos essenciais na elaboração de problemas mal estruturados são a força direcionadora da investigação.

A realidade é que os alunos que são novos para o $\mathrm{ABP}$ necessitam de suportes educacionais para apoiar o desenvolvimento de habilidades de resolução de problemas, habilidades de aprendizagem autodirigidas e trabalho em equipe/colaboração até atingirem um nível de autossuficiência, no qual esses suportes podem ser removidos (Kiili, 2007). $\mathrm{O}$ desafio de muitos instrutores quando adotam a abordagem ABP, é realizar a transição de professor, como fornecedor de conhecimento, para tutor, como gerente e facilitador do aprendizado (Ertmer e Simons, 2006). Hmelo-Silver (2004) argumenta que o ABP é elaborado de forma a ajudar os estudantes a:

- Construir uma base de conhecimento ampla e flexível;

- Desenvolver efetivas habilidades de resolução de problemas;

- Desenvolver habilidades de aprendizagem autodirigidas e que durem ao longo da vida; e
- Tornarem-se intrinsecamente motivados para aprender.

Além de todas as características citadas até aqui, ainda existem, de acordo com Yeo (2005), três fatores críticos que influenciam a resposta do aluno ao ABP:

- Mentalidade: em qualquer jornada de aprendizado, a correta maneira de pensar é o principal motivo para obtenção e aplicação do conhecimento;

- Avaliação do progresso: ao contrário de métodos convencionais, o ABP não pode ser avaliado apenas através de testes ou exames, mas sim com uma variedade de métodos. A variedade contribui para garantir que cada estágio do aprendizado foi atingido em nível satisfatório, de forma que os alunos desenvolvam seu processo cognitivo em busca de possíveis soluções para o problema em estudo; e

- Dinâmica humana: é através da autoaprendizagem que o compartilhamento de conhecimentos em grupo se torna importante para o aluno. Aprendizagem compartilhada depende muito da dinâmica de interação dos alunos.

\section{METODOLOGIA}

Seguindo o ciclo de planejamento/ação/observação/reflexão (Hung, 2009), a primeira etapa do trabalho foi de avaliar como um problema relacionado à vida real poderia ser aplicado em sala de aula. A partir deste ciclo inicial, o pesquisador e o docente avaliariam como estabelecer a aplicação do método ABP durante a disciplina de Logística e Transportes. As opções para aplicação do primeiro ciclo seriam para uma das seguintes turmas: graduação em Administração de Empresas graduação em Engenharia de Produção ou mestrado em Engenharia de Produção. A escolha foi pela aplicação no mestrado. O principal elemento desta escolha foi o fato de que os alunos do mestrado poderiam contribuir de forma mais relevante e participativa para a avaliação em todas as etapas, visto que além de alunos são também pesquisadores "em formação". Além disso, naquele momento da pesquisa haveria flexibilidade em termos de carga horária do docente, dos alunos e do pesquisador para realização do teste em sala de aula.

A proposta inicial do problema ABP utilizado na pósgraduação foi a seguinte: "Um empresário da região do Sul de Minas está interessado em investir na fabricação de bicicletas, inicialmente nos modelos definidos como bicicleta de montanha e vendê-las para algumas regiões do Brasil. A análise da viabilidade inicial deste investimento mostrou-se interessante, porém não há dados suficientes sobre o processo de compra dos componentes dos fabricantes nacionais e estrangeiros. Estes componentes são divididos em 12 famílias (relacionadas a cada parte da bicicleta, Figura 1) e pelo menos 31 categorias (componentes de cada família). Cada categoria possui um número elevado de opções relacionadas a custos, modelos, fabricantes e origens (mapa da Figura 1), tornando extensa a lista de itens possíveis para compra extensa. A experiência deste empresário é limitada neste aspecto e ele contratou uma consultoria (os alunos) para auxiliá-lo."

A proposta é que o problema seja resolvido em grupos. $\mathrm{O}$ formato de trabalho das reuniões seguiu um modelo pré- 


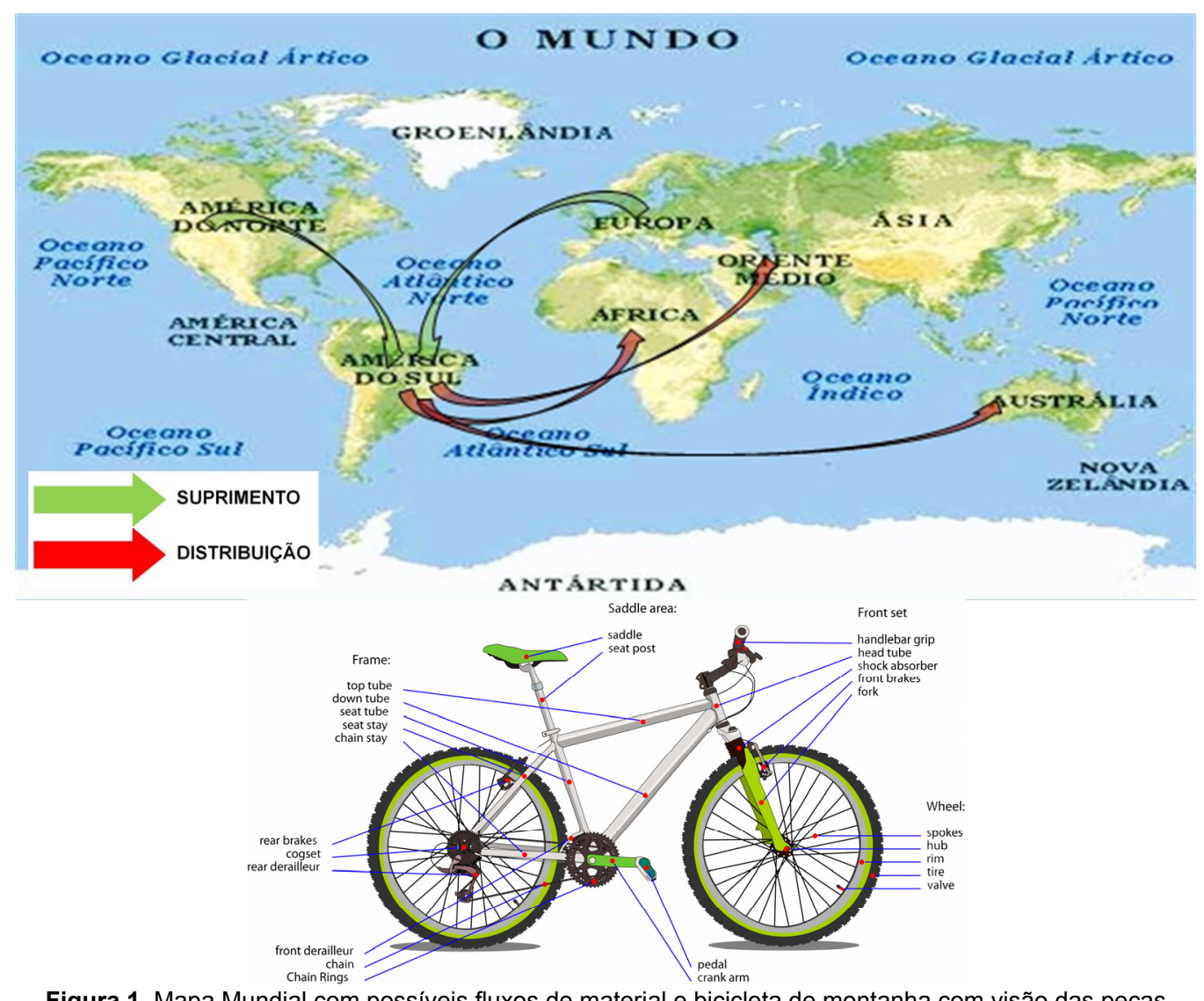

Figura 1. Mapa Mundial com possíveis fluxos de material e bicicleta de montanha com visão das peças

especificado, porém simples. Em cada reunião da equipe há um líder que se encarrega de manter o trabalho focado. Os membros da equipe alternam as funções em cada reunião, incluindo o líder da reunião e o secretário. Para cada problema atribuído, as equipes devem preparar um relatório escrito e uma apresentação oral, como forma de avaliação. Além disso, o docente e o pesquisador avaliam o trabalho apresentado pelos alunos e debatem com os mesmos as dificuldades encontradas, os erros e acertos de conceitos teóricos, além dos resultados e propostas apresentadas. O método de avaliação foi realizado por meio de observação do pesquisador e do docente, entrevistas (perguntas abertas) e um questionário estruturado (perguntas fechadas) com escala de concordância do tipo Likert submetida à análise da confiabilidade (coeficientes alfa de Cronbach), da validade (Análise Fatorial) e da regressão, que buscam - utilizandose de métodos estatísticos - traduzir em números, as opiniões, sentimentos e informações. Para validar o objetivo da pesquisa utilizou-se o método proposto por Kanet e Barut (2003), através das hipóteses adaptadas descritas na Tabela 1 .

Para testar estas hipóteses, será utilizado também o modelo descrito por Kanet e Barut (2003), com as adaptações necessárias para o curso de Logística e Transportes. Tratam-se de 37 variáveis, em formato de questionário, que mediram os fatores apresentados. Estes, por sua vez, definem a efetividade do estudo em questão.

Seguindo a descrição fornecida, foi realizado o exercício em sala de aula no curso de Logística Empresarial para o mestrado em Engenharia de Produção. A atividade teve duração de três semanas, período em que as aulas presenciais aconteceram uma vez por semana. Foi explicado aos alunos o conceito de ABP e todos foram avisados que se tratava de um exercício, fase de um primeiro ciclo para uma pesquisa de mestrado. O problema da fábrica de bicicletas foi aplicado, restrito ao uso dos conceitos de transportes unimodais e intermodais. O professor da disciplina utilizou-se do mesmo material usado tradicionalmente para explicar os conceitos desse tema, porém o tempo utilizado foi cerca de um quarto das aulas expositivas regulares. O restante do tempo foi utilizado para explicar os conceitos do ABP, separar grupos, explicar a forma de trabalho com atas e reuniões obrigatórias. Além disso, os alunos usaram o tempo em sala para realizar algumas atividades práticas. Também foi solicitado aos alunos uma reunião extra (fora da aula) para avaliar organização e conciliação de agendas.

O professor e o pesquisador ficaram $100 \%$ do tempo presentes em sala para esclarecimento de dúvidas e observação dos trabalhos das equipes. O pesquisador se colocou à disposição fora do horário das aulas esclarecimento de dúvidas

Tabela 1. Hipóteses e as correspondências de seus fatores

\begin{tabular}{lll}
\hline & \multicolumn{2}{l}{$\boldsymbol{F 3 - \text { Aderência ao Método ABP }}$} \\
\cline { 2 - 3 } & $H 0:$ & $H 1$ : \\
\hline F1 - Conhecimento em Logística e Transportes & Não há relação entre F3/F1 & Há relação entre F3/F1 \\
F2 - Resolução de problemas / comunicação / & & \\
desenvolvimento de habilidades & Não há relação entre F3/F2 & Há relação entre F3/F2 \\
F4 - Confiança / Satisfação do estudante & Não há relação entre F3/F4 & Há relação entre F3/F4 \\
\hline
\end{tabular}


sobre o método ou atividades. Ao final das três semanas foi aplicado um questionário para os alunos avaliarem sua participação e opinarem sobre a metodologia. No último dia de atividades, durante as apresentações finais dos trabalhos, os alunos fizeram observações sobre os resultados das outras equipes e comentários sobre as dificuldades encontradas. Além disso, após as apresentações, os alunos realizaram um pequeno debate com o pesquisador para apresentar comentários, percepções individuais e sugestões para a aplicação do método ABP em sala de aula.

\subsection{A aplicação na graduação}

Seguindo a mesma linha da proposta de problema, foram elaborados temas para os problemas da graduação com objetivos claros: Estacionamento (introdução dos conceitos do ABP - não exigia conhecimento prévio), Gerenciamento da cadeia de suprimentos, Gestão de Relacionamento e Nível de Serviço Logístico, Canais de Distribuição e Transportes e Modais. Esses problemas foram elaborados para aplicação, dentro da disciplina de Logística e Transporte, nos cursos de Engenharia de Produção e Administração de Empresas. A distribuição do tempo entre apresentação da teoria e trabalhos práticos pelos alunos em sala de aula foi definida em conjunto pelo docente e pelo pesquisador. A proposta, segundo as possibilidades apresentadas por Moesby (2008), foi de uma carga horária de aproximadamente $60 \%$ de teoria e $40 \%$ dedicada para trabalhos práticos desenvolvidos pelos alunos.

\section{RESULTADOS E DISCUSSÕES}

Os resultados e a discussão dos mesmos foram pautados em análises qualitativas e quantitativas, apresentadas nos subtópicos a seguir.

\subsection{Análise qualitativa}

Ao final de cada ciclo da pesquisa foi realizado um breve debate, sem a presença do docente, na qual se procurou i- dentificar junto aos alunos os aspectos positivos e negativos do método, que aparecem sintetizados na Tabela 2.

Foram ainda extraídos do debate com os estudantes diversos comentários a respeito do uso do conceito de ABP, reproduzidos sucintamente a seguir:

- Seu uso depende muito da disciplina, da matéria a ser ensinada;

- Seria interessante a adaptação em outras matérias;

- A adaptação de todo o curso em função deste método, para integrar o ensino e o uso das disciplinas de forma inter-relacionada, deve ser considerada;

- O problema mal estruturado era "muito mal estruturado". O professor poderia definir uma resposta clara e objetiva para os problemas apresentados;

- Usar o método de ABP já como avaliação de aproveitamento, sem a necessidade de entrega de artigos ou provas de conhecimento;

- A avaliação dos trabalhos realizada pelo professor e pelo pesquisador foi considerada melhor no último dia. Isto porque optou-se por realizar um comentário único sobre todas as apresentações realizadas durante a disciplina, além de comentários gerais sobre a aplicação do método no geral;

- O preenchimento de atas não foi bem recebido, pois não percebeu-se valor na atividade, considerando-a como tomadora de tempo (alguns chegaram a declarar que $80 \%$ do tempo do trabalho era dedicado ao preenchimento de atas);

- Consideraram difícil realizar uma reunião por semana fora do horário de aula;

- Sentiram falta de foco, no sentido de buscar o quê exatamente fazer. O problema mal estruturado e a falta de perguntas objetivas a serem respondidas, como num questionário, dificultaram o andamento dos trabalhos;

- Os alunos pediram que o retorno das notas dos relatórios fosse acompanhado de comentários, como por exemplo, o que faltou em termos de conteúdo ou

Tabela 2. Percepção dos alunos sobre a aplicação do método

\begin{tabular}{|c|c|}
\hline Aspectos Positivos: & Aspectos Negativos: \\
\hline $\begin{array}{l}\text { - Fixação dos conceitos teóricos e práticos do tema mais eficaz } \\
\text { do que o método tradicional de ensino (nesta observação e na } \\
\text { anterior os alunos usaram como referência os temas e as aulas } \\
\text { assistidas antes da aplicação do método ABP); } \\
\text { - Investigação com foco mais prático para a solução do } \\
\text { problema; } \\
\text { - Maior comprometimento dos participantes para solução do } \\
\text { problema; } \\
\text { - Desenvolvimento de trabalho em equipe; } \\
\text { - Maior preocupação em pesquisar assuntos relevantes para a } \\
\text { solução do problema; } \\
\text { - Proporciona uma visão geral da disciplina e de seu uso na } \\
\text { prática, em situações do mundo real. Desta forma, acreditam } \\
\text { que haverá maior retenção de conhecimento e conexão dos } \\
\text { conceitos às aplicações; } \\
\text { - Os conceitos introduzidos ao longo do processo auxiliavam } \\
\text { na coleta de informação; } \\
\text { - O trabalho em grupo, que permite conhecer melhor e trocar } \\
\text { ideias com outros alunos; } \\
\text { - Maior retenção dos conceitos devido à resolução de } \\
\text { problemas na prática; } \\
\text { A relevância de algumas informações entre todos os dados } \\
\text { pesquisados fica evidenciada, conduzindo ao que é mais } \\
\text { importante. }\end{array}$ & $\begin{array}{l}\text { - Tempo para solução do problema e número de reuniões } \\
\text { insuficientes; } \\
\text { - Pouca comunicação com os tutores; } \\
\text { - Dificuldade para encontrar disponibilidade de todos os } \\
\text { participantes fora do horário de aula; } \\
\text { - Desperdício de tempo pesquisando assuntos irrelevantes } \\
\text { para a solução do problema; } \\
\text { - Falta de uma estrutura pré-definida na elaboração das atas } \\
\text { das reuniões; } \\
\text { - Falta de atividades mais práticas, como uma visita a uma } \\
\text { empresa no início das aulas para elaborar a descrição de } \\
\text { um problema real e resolvê-lo ao final da disciplina; } \\
\text { - A obrigatoriedade do papel do líder e do secretário foi } \\
\text { considerada uma "formalidade", não sendo sempre } \\
\text { desempenhada; } \\
\text { - Dificuldades geradas pela interferência de trabalhos } \\
\text { solicitados por outras disciplinas e processos de seleção } \\
\text { de estágio; } \\
\text { - A quantidade de dados e informações disponibilizados foi } \\
\text { considerada insuficiente por alguns alunos para a } \\
\text { realização de cálculos. }\end{array}$ \\
\hline
\end{tabular}


formatação;

- Após as apresentações, os estudantes gostariam de conhecer a "melhor resolução" do problema sob a perspectiva do professor ou, se possível, como uma empresa resolveu um problema similar na vida real;

- A busca de informação real e da liberdade de ação para escolher o caminho a seguir e foi algo novo para os alunos. Isso permitia que as soluções das outras equipes fossem vistas como oportunidades de analisar o problema sob uma perspectiva diferente;

- Sobre a apresentação dos trabalhos, alguns consideraram "cansativo" escutar todos os grupos apresentarem o mesmo tema. Uma sugestão seria todos prepararem o trabalho, mas apenas alguns apresentarem, com critério de sorteio a ser definido pelo docente; e

- Alguns alunos sugeriram alterações na ementa da disciplina, como a inclusão do tema "Comércio Internacional".

Já pela perspectiva do docente e do pesquisador, destacam-se a seguir as principais observações realizadas ao longo dos ciclos da pesquisa:

- O ABP aumenta a imprevisibilidade em sala de aula, associada à possibilidade do levantamento de questões conceituais desconhecidas para o docente. A possibilidade dos alunos tomarem rumos diferentes em suas pesquisas também se enquadra nesta observação, o que dificulta a cobertura dos conteúdos planejados para o componente curricular. Em razão disso, o docente acredita que o domínio do conteúdo e a experiência em sala de aula são importantes para uma atuação bem sucedida do método.

- Durante as avaliações, o professor e o pesquisador não consideraram se a solução proposta era boa ou ruim, correta ou errada, mas sim as justificativas e a linha de raciocínio utilizada;

- Houve pouca procura dos alunos ao professor para esclarecer dúvidas, da teoria ou da prática. Havia um horário semanal, já combinado e comunicado aos alunos no início da disciplina, exclusivamente para esclarecimento de dúvidas - pouquíssimos alunos o utilizaram. Isso foi relatado posteriormente aos alunos, quando ao final sugeriram que o retorno das notas dos relatórios parciais fosse acompanhado de comentários;

- Os alunos não consideraram a importância de se destacar o objetivo nas atas das reuniões como um direcionador para investigação da solução do problema proposto;

- Para os temas relacionados a transportes, os alunos praticamente não apresentaram cálculos ou métodos quantitativos para justificar suas argumentações;

- Durante as apresentações, os alunos citaram vários conceitos relacionados à disciplina de Logística e Transportes, mas não usaram conceitos de outras disciplinas conforme era esperado pelo professor (tais como avaliação de custos, gestão de estoques, lead time etc.); e

- Os alunos aprenderam a respeitar o horário estipulado para cada apresentação ao longo da disciplina (no último dia quase $100 \%$ dos grupos apresentaram dentro do limite de tempo).

Com relação às diferenças observadas entre a aplicação em Engenharia e Administração, pode-se concluir que, de maneira geral, os trabalhos apresentados pela turma da Administração mostraram uma tendência de tratar mais teoria e conceitos do que números ou cálculos, quando comparado aos trabalhos apresentados pela Engenharia de Produção. Outras observações são sintetizadas na Tabela 3.

\subsection{Análise quantitativa}

Do ponto de vista quantitativo, para verificar a efetividade do método ABP utilizou-se do modelo proposto por Kanet e Barut (2003), com o emprego da Análise Fatorial (técnica multivariada), do Alfa de Cronbach (para checar a confiabilidade dos dados) e da Regressão Linear Simples (para verificar as hipóteses da pesquisa). Informações mais detalhadas sobre as análises estatísticas efetuadas podem ser encontradas em Cardoso (2011).

Conforme Hair Jr. et al. (2009), a Análise Fatorial é uma "técnica de interdependência na qual todas as variáveis são simultaneamente consideradas, cada uma relacionada com todas as outras, empregando ainda o conceito da variável estatística, a composição linear de variáveis". Para os autores, a Análise Fatorial pode atingir seus objetivos nas perspectivas exploratória ou confirmatória. Eles explicam que, na Análise Fatorial Exploratória, o pesquisador "analisa, entende e identifica uma estrutura de relacionamento entre as variáveis a partir do resultado da Análise Fatorial".

O teste Kaiser-Meyer-Olkin (KMO) compara as correlações simples com as parciais observadas entre as variáveis e indica, segundo Pestana e Gageiro (2003), o grau de explicação dos dados a partir dos fatores encontrados na Análise Fatorial. Estes autores ainda apresentam os valores para interpretar o KMO: muito boa - entre 1 e 0,9 ; boa - entre 0,8 e 0,9 ; média - entre 0,7 - 0,8 ; razoável - entre 0,6 e 0,7 ; má - entre 0,5 e 0,6 ; inaceitável - menor que 0,5 , pois indicam que a realização da análise fatorial é insatisfatória devido à correlação fraca entre as variáveis.

De acordo com Hair Jr. et al. (2009), o teste de esfericidade de Bartlett (Bartlett's Test of Sphericity - BTS) é “o

Tabela 3. Observações entre os alunos de graduação em Engenharia e Administração

\begin{tabular}{ll}
\hline Engenharia & Administração \\
\hline Mais focado, mais exato & Mais abrangente \\
Cálculos e métodos claros & Mencionam outras disciplinas e integram mais temas \\
Limitam-se a resolver o problema apresentado & Discutem marketing e oportunidades não avaliadas \\
Reuniões de trabalho em sala de aula & Problemas com a carga horária (pouco tempo para apresentações) \\
Dificuldade em formular hipóteses & Dificuldade em formular hipóteses \\
Como estimular o uso de outros conhecimentos? & Preocupação demasiada com ata de reunião \\
& Como adaptar melhor o método para a carga horária disponível? \\
\hline
\end{tabular}


teste estatístico da significância geral de todas as correlações em uma matriz de correlação". Este teste indica se existe relação suficiente entre as variáveis para a aplicação da Análise Fatorial, sendo recomendado um valor de Significância menor que 0,05. Caso o valor da Significância alcance 0,10, o uso da Análise Fatorial é desaconselhável. Ainda segundo Hair Jr. et al. (2009), o Measure of Sampling Adequacy (MSA) é a medida de avaliação da adequação da amostra, a qual tem um intervalo inadmissível se abaixo de 0,50. O MSA encontra-se na diagonal da Matriz Anti-imagem. As comunalidades representam a proporção de variância explicada pela solução fatorial para cada variável (Pestana e Gageiro, 2003). As variáveis conseguem alto poder de explicação, considerando-se todos os fatores, se superiores a 0,70 (Hair Jr. et al., 2009).

Para verificar a confiabilidade dos escores fatoriais dos fatores retidos, utiliza-se o Alfa de Cronbach. A análise de confiabilidade dos dados permite determinar a extensão em que os itens estão relacionados com os demais. O Alfa de Cronbach é uma das medidas de consistência interna mais utilizada. Assim, de acordo com Pestana e Gageiro (2003), o Alfa de Cronbach verifica a "consistência interna de um grupo de variáveis (itens), podendo definir-se como a correlação que se espera obter entre a escala usada e outras escalas hipotéticas do mesmo universo, com igual número de itens, que meçam a mesma característica". Para os autores, o Alfa negativo não viola o modelo e nem inviabiliza o seu uso. Eles consideram a consistência como: (i) Muito boa $=>$ alfa superior a 0,9 ; (ii) Boa $=>$ alfa entre 0,8 e 0,9 ; (iii) Razoável $\Rightarrow$ alfa entre 0,7 e 0,8 ; (iv) Fraca $=>$ alfa entre 0,6 e 0,7 ; e (v) Inadmissível $\Rightarrow>$ alfa menor que 0,6. Hair Jr. et al. (2009) consideram o limite inferior de aceitabili-

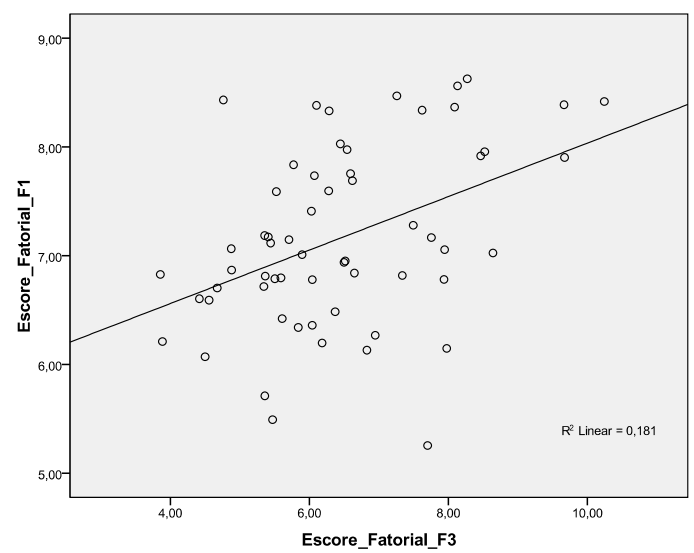

dade para o Alfa de Cronbach os valores de 0,60 e 0,70.

Conforme o modelo proposto por Kanet e Barut (2003), a efetividade é verificada por meio da Regressão. Assim, emprega-se a Regressão com o intuito de atingir o objetivo da pesquisa e verificar a efetividade do método ABP por meio dos escores fatoriais obtidos na análise fatorial. $\mathrm{O}$ valor $\mathrm{P}$ (ou valor de probabilidade), segundo Levine et al. (2000), é a probabilidade de se obter um valor da estatística de teste que seja no mínimo tão extremo quanto o que representa os dados amostrais, supondo que a hipótese nula seja verdadeira. A hipótese nula é rejeitada se o valor $\mathrm{P}$ for muito pequeno, tal como 0,05 ou menos.

Considerando os questionários respondidos, os dados da amostra referem-se a 60 respondentes, sendo 51,67\% do gênero masculino e 48,33\%, do feminino. São divididos em $56,67 \%$ no curso de Administração e 43,33\% no curso de Engenharia de Produção. Quanto à idade, mais de 78\% possuem entre 21 e 23 anos. Foi realizada a análise fatorial com o propósito de reduzir o número de variáveis em escores fatoriais para uso posterior na regressão. Determinou-se o uso de um fator utilizando-se as variáveis do Fator F1 (Conhecimento em Logística e Transportes) proposto no modelo. Em seguida, o mesmo procedimento foi realizado para a busca de um fator para as variáveis do Fator F2 (Resolução de problemas / comunicação / desenvolvimento de habilidades), do Fator F3 (Aderência ao método ABP) e do Fator F4 (Confiança / Satisfação do estudante). A confiabilidade foi medida pelo Alfa de Cronbach, o qual foi calculado separadamente, da mesma forma que a análise fatorial, para que fosse medida a consistência das variáveis de cada fator proposto no modelo. Observam-se os Diagramas de Dispersão da Figura 2 para “analisar o comportamento das

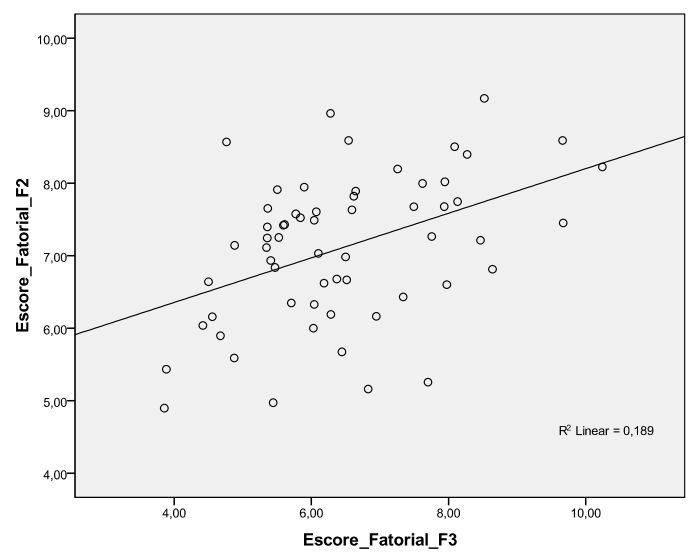

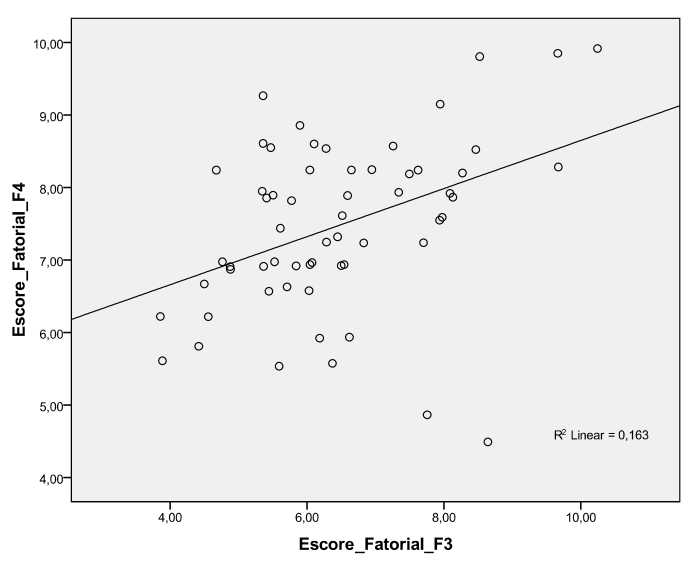

Figura 2. Diagrama de Dispersão F3-F1, F3-F2 E F3-F4 
variáveis e se ter uma ideia da relação existente entre as mesmas" (Levine et al., 2000). Notam-se os pontos que se concentram e os que se desviam da reta sugerindo uma relação linear positiva entre as variáveis F3-F1, F3-F2 e F3F4. Aceita-se que uma das duas hipóteses a seguir é verdadeira:

- H0: P = 0 (ou seja, F3 não é correlacionado com os outros fatores F1, F2 e F4); e

- H1 : $\mathrm{P} \neq 0$ (ou seja, F3 é correlacionado com os outros fatores F1, F2 e F4).

Todos os resultados do $p$-value foram menores que 0,05 , o que leva a rejeitar H0: $\mathrm{P}=0$ (HAIR JR. et al., 2009). Assim, há evidência para apoiar a afirmativa de que o fator F3 está correlacionado com os fatores F1, F2 e F4. Foram utilizados os escores fatoriais extraídos da análise fatorial para realizar a Regressão Linear Simples. O Escore Fatorial, segundo Hair Jr. et al. (2009), é uma medida composta para cada observação sobre cada fator extraído na análise fatorial. Segundo os autores, o escore fatorial pode ser utilizado para representar o(s) fator(es) em análises subsequentes. Como o objetivo geral do estudo é verificar a efetividade do método $\mathrm{ABP}$, foi realizada uma regressão para cada um dos pares de constructos. Para considerar válida a hipótese proposta, verificou-se a distribuição t de Student e o valor $\mathrm{P}$ obtido em cada um dos casos, decompondo desse modo a hipótese inicial em três, estabelecidas a seguir:

- Para a Regressão do Fator F3 com o Fator F1 (do Fator F3 com o Fator F2 / do Fator F3 com o Fator F4):

- H0: $\mathrm{A}=0$; indica que a reta de regressão passa na origem;

- H1: A $\neq 0$; indica que a reta de regressão não passa na origem;

- H0: B = 0; indica que o coeficiente F1 (F2/F4) é zero, não correlacionando com F3; e

- H1: B $\neq 0$; indica que o coeficiente F1 (F2/F4) não é zero, correlacionando com F3.

Com os resultados encontrados para a verificação do coeficiente linear A, rejeita-se H0: A $=0$ nas três Regressões, pois não há evidências suficientes para se concluir que a reta de regressão passa na origem. Para sustentar essa afirmação, tem-se que os valores encontrados do $p$-value foram todos inferiores a 0,05 , o que leva a rejeitar a hipótese nula H0 (HAIR JR. et al., 2009). Para o coeficiente angular B, todos os valores de p-value também foram inferiores a 0,05 , o que leva a rejeitar a hipótese nula $\mathrm{H} 0$ : $\mathrm{B}=0$, ou seja, os coeficientes F1, F2 e F4 são correlacionados com o coeficiente F3. Observou-se em seguida a análise gráfica dos resíduos, que é a diferença entre o valor observado e o va- lor projetado pela regressão, onde nota-se a ausência de autocorrelação residual, já que os resíduos se distribuem aleatoriamente, não se concentrando acima ou abaixo da reta horizontal. Com isso pode-se deduzir que a reta de regressão é uma boa representação dos dados observados, ou seja, os valores obtidos "esperados" através da equação de regressão linear estão bastante próximos dos valores "observados" na prática.

\subsubsection{Análise do Fator 4 revisado}

A sequência a seguir foi aplicada para todos os fatores F1 a F4, como parte da análise quantitativa, e um resumo dos resultados pode ser observado na Tabela 4. O fator F4, entretanto, necessitou de revisão. Para verificar a consistência dos dados foi aplicado o teste de KMO, apresentando um índice igual a 0,535 , indicando uma possível correlação fraca entre as variáveis. Na realização do teste BTS verificou-se que é improvável a matriz de correlação ser uma identidade pelo nível de significância igual a zero, possibilitando ainda a aplicação da análise fatorial.

O MSA inicialmente encontrado estava inferior a 0,5 (encontrado valor de 0,447 na variável B20), que é considerado um resultado inaceitável, pois está abaixo do limite de 0,5 . As comunalidades variavam de 0,069 a 0,414 . Optouse por excluir a variável B20 (a questão "Ser desafiado para esclarecer as próprias opiniões"), devido ao fato de ser a única com o valor MSA abaixo de $0,5(0,447)$ e com a comunalidade mais baixa de todas $(0,069)$, e repetir os testes para averiguar se há alguma melhora nos resultados. Foi aplicado o teste de $\mathrm{KMO}$, apresentando um índice igual a 0,547 , resultado pouco melhor que o anterior. Na realização do teste BTS o nível de significância permaneceu igual a zero. O MSA está superior a 0,505, o que é considerado aceitável. As comunalidades variam de 0,295 a 0,469, o que não demonstra uma forte relação com o fator $\mathrm{F} 1$, sendo consideradas relações apenas razoáveis.

Para as variáveis do fator F4 obteve-se um valor para o Alfa de Cronbach de 0,516, valor considerado fraco, antes de retirar a variável B20. Após a retirada da variável B20, o valor encontrado para o Alfa de Cronbach foi de 0,544. Em alguns cenários de investigação das ciências sociais, um Alfa próximo de 0.60 é considerado aceitável desde que os resultados obtidos com esse instrumento sejam interpretados com precaução (Maroco e Garcia-Marques, 2006). Ainda segundo Maroco e Garcia-Marques (2006), o valor de fiabilidade estimado pelo Alfa de Cronbach não é uma característica de um instrumento, mas uma estimativa da fiabilidade dos dados obtidos que podem informar sobre a precisão do instrumento. Desta forma, através da regressão em conjunto com todos os dados quantitativos obtidos anteriormente, pode-se afirmar que o fator F3 (Aderência ao Méto-

Tabela 4. Abordagem quantitativa: Análise Fatorial, Alfa de Cronbach e Correlação

\begin{tabular}{lllllll}
\hline Teste & $\begin{array}{l}\text { Faixa } \\
\text { Aceitável }\end{array}$ & $\boldsymbol{F 1}$ & $\boldsymbol{F 2}$ & $\boldsymbol{F 3}$ & $\boldsymbol{F 4}$ & $\begin{array}{l}\boldsymbol{F 4} \\
\text { revisado }\end{array}$ \\
\hline KMO & $>0,50$ & 0,817 & 0,770 & 0,724 & 0,535 & 0,547 \\
BTS & $<0,05$ & 0 & 0 & 0 & 0 & 0 \\
MSA & $>0,50$ & 0,723 & 0,620 & 0,620 & 0,447 & 0,505 \\
$\alpha$ Cronbach & $>0,60$ & 0,904 & 0,821 & 0,821 & 0,516 & 0,544 \\
Correlação com F3 & $<0,05$ & 0,001 & 0,001 & & & 0,001 \\
\hline Sendo: KMO - Teste Kaiser-Meyer-Olkin & & & &
\end{tabular}

Sendo: KMO - Teste Kaiser-Meyer-Olkin

BTS - Teste de esfericidade de Bartlett (Bartlett's Test of Sphericity)

MSA - Measure of Sampling Adequacy, ou Medida de Adequação da Amostra. 
do ABP) influencia as respostas F1 (Conhecimento em Logística e Transportes), F2 (Resolução de problemas / comunicação / desenvolvimento de habilidades) e F4 (Confiança / Satisfação do estudante), dados estes que se mostraram consistentes com os resultados da hipótese de relacionamento entre os fatores F3-F1, F3-F2 e F3-F4 obtidos pelo modelo de Kanet e Barut (2003).

\section{CONCLUSÕES}

Seguramente, não se pode afirmar que há um método que resolva todos os problemas de ensino-aprendizagem e nem tampouco um método que garanta colocação no mercado de trabalho e sucesso na vida profissional, mas há alguns métodos que podem promover a satisfação da maioria dos estudantes. $\mathrm{O}$ aprendizado baseado em problemas pode auxiliar os estudantes ao colocar conhecimento e aprendizagem em um contexto do mundo real, o que permite a eles agirem como profissionais e se prepararem melhor para o mercado de trabalho. Com isso em mente, esta pesquisa teve sua origem na proposta de analisar a efetividade da aprendizagem baseada em problema nos cursos de Engenharia de Produção e Administração de Empresas, durante a disciplina de Logística e Transportes na Universidade Federal de Itajubá. As análises foram feitas do ponto de vista quantitativo e qualitativo.

Quantitativamente, confirmou-se a hipótese de existência de relacionamento entre os fatores F3 (aderência ao Método ABP) e os demais fatores F1 (Conhecimento em Logística e Transportes), F2 (Resolução de problemas / comunicação / desenvolvimento de habilidades) e F4 (Confiança / Satisfação do estudante) através da aplicação de questionário para os alunos e da análise quantitativa (Análise Fatorial, Alfa de Cronbach, Correlação e Regressão) dos dados obtidos. A análise de resíduos comprovou que as equações de F3F1, F3-F2 e F3-F4, obtidas através da regressão, foram consideradas boas representações dos dados observados.

Qualitativamente, os resultados da pesquisa mostraram a efetividade do método através das observações dos alunos, do pesquisador e do docente. Os alunos consideraram a aula com este método mais dinâmica e motivadora que as aulas expositivas. Registraram que o ABP promove uma visão diferente sobre uma mesma situação, ao ter a oportunidade de avaliar os trabalhos apresentados por seus colegas, e a integração entre a teoria e a prática. As habilidades desenvolvidas mais citadas foram relacionadas ao estudo autônomo, convívio em grupo e a busca por informações que sejam de fato relevantes durante uma pesquisa. Por outro lado, os alunos reconheceram que o ABP demanda mais tempo, o que conflita com outras atividades relativas aos demais componentes curriculares e estágios.

$\mathrm{O}$ docente considerou a aula com o ABP mais dinâmica e desafiadora para si e para os alunos, pois aumenta a imprevisibilidade em sala de aula, associada à possibilidade do levantamento, por parte dos alunos, de questões conceituais desconhecidas para o docente. A possibilidade dos alunos tomarem rumos diferentes em suas pesquisas também se enquadra nesta observação, fatores que dificultam a cobertura dos conteúdos planejados para o componente curricular. Em razão dessa imprevisibilidade, o docente acredita que o domínio do conteúdo e a experiência em sala de aula são atributos importantes para uma atuação bem sucedida do método.
É possível concluir que a eficácia do método $\mathrm{ABP}$ está diretamente relacionada com o preparo e adaptação dos professores e alunos, face às peculiaridades do método. Outro ponto é a necessidade de um maior cuidado do docente na explicação do funcionamento do novo método para os alunos (como sugestão, adotar um problema mais lúdico para mostrar o processo de funcionamento do ABP). O docente também precisa ter em conta que o objetivo do método é melhorar a qualidade do aprendizado do aluno e não da qualidade do ensino do professor. Isto leva a uma demanda consideravelmente maior de tempo em processos de preparação de problemas, atendimentos individuais e aconselhamento do que na preparação de slides para apresentação em sala de aula.

O fato de que problemas reais, ou próximos dos reais, podem ser cativantes na universidade de uma forma que livros ou apostilas não são é, sem dúvida, um recurso a ser utilizado pelos profissionais de educação. Entretanto, devese moderar o entusiasmo, com o conhecimento de que problemas com objetivos educacionais e motivadores precisam ser cuidadosamente construídos para fornecer uma experiência atraente, bem como suportar o aprendizado adequado ao aluno.

Embora esteja além desta pesquisa discutir a validade e a propriedade de avaliações tradicionais (como, por exemplo, as provas individuais ao longo da disciplina), tidas como objetivas, é preciso ressaltar que a sistemática de avaliação de rendimento dos alunos no ABP deve ser obrigatoriamente coerente com seus princípios. Se isso não acontecer, há o risco de mensagens conflitantes com relação aos objetivos educacionais serem passadas aos alunos, ou seja, a avaliação do aluno também é um aspecto importante e deve ser mantido.

Deve-se levar em consideração também que o ponto controverso do aprendizado baseado em problemas não está somente nos métodos ou na dificuldade de avaliar o conhecimento ganho, mas também na inabilidade do ser humano em traduzir seu conhecimento em ações positivas e benéficas, seja na simulação de um problema ou na vida real. Assim, não se espera que a abordagem apresentada nesse trabalho represente um processo concluído. Ao contrário, o trabalho deve ser percebido como o retrato do início de um processo contínuo de aprendizado e de desenvolvimento, com posteriores refinamentos.

\section{AGRADECIMENTOS}

Os autores agradecem ao $\mathrm{CNPq}$ (Conselho Nacional de Desenvolvimento Científico e Tecnológico) e a FAPEMIG (Fundação de Amparo à Pesquisa do Estado de Minas Gerais), pelo apoio financeiro concedido a diversos projetos que subsidiaram o desenvolvimento desse trabalho.

\section{REFERÊNCIAS BIBLIOGRÁFICAS}

Cardoso, I. M. (2011) Métodos ativos de aprendizagem: o uso do aprendizado baseado em problemas no ensino de logística e transportes. Dissertação (Mestrado). Programa de Pós-Graduação em Engenharia de Produção, Universidade Federal de Itajubá, Itajubá.

Crookall, D. e W. Thorngate (2009) Acting, Knowing, Learning, Simulating, Gaming (Editorial). Simulation \& Gaming, v. 40, n. 1, p. 826. DOI: $10.1177 / 1046878108330364$.

Ertmer, P. A. e K. D. Simons (2006). Jumping the PBL implementation hurdle: Supporting the efforts of $\mathrm{K}-12$ teachers. Interdisciplinary Journal of Problem-based Learning, v.1, n. 1, p. 40-54. DOI: 10.7771/1541-5015.1005.

Hair Jr., J. F; W. C. Black; B. J. Babin e R. E. Anderson (2009) Multivariate Data Analysis. Prentice Hall, $7^{\mathrm{a}}$ Edição. 816 p. 
Hmelo-Silver, C. E. (2004) Problem-Based Learning: What and How Do Students Learn? Educational Psychology Review, v. 16, n. 3, p. 235-266. DOI: 10.1023/B:EDPR.0000034022.16470.f3.

Hung, W. (2009) The 9-step problem design process for problem-based learning: Application of the 3C3R model. Educational Research Review, v. 4, n. 2, p. 118-141. DOI: 10.1016/j.edurev. 2008.12.001.

Kanet, J. J. e M. Barut (2003) Problem-Based Learning for Production and Operations Management. Decision Sciences Journal of Innovative Education, v. 1 n. 1, p. 99-118. DOI: 10.1111/1540-5915.00007.

Kiili, K. (2007) Foundation for problem-based gaming. British Journal of Educational Technology, v. 38, n. 3, p. 394-404. DOI: 10.1111/j.1467-8535.2007.00704.x.

Kuri, N. P.; G. G. Manzato e A. N. R. Silva (2007) Aprendizado baseado em problemas em uma plataforma de ensino a distância: uma aplicação do COL na EESC-USP. Revista Minerva, v. 4, p. 27-39.

Kuri, N. P. e A. N. R. Silva (2010) Uma estratégia de ensino em transportes apoiada nos perfis de personalidade dos estudantes. Transportes, v. 18, n. 3, p. $72-79$.

Levine, D. M.; M. L. Berenson e D. Stephan (2000) Estatística: Teoria e Aplicações. Livros Técnicos e Científicos Editora AS, Rio de Janeiro.

Maroco, J. e T. Garcia-Marques (2006) Qual a fiabilidade do alfa de Cronbach? Questões antigas e soluções modernas. Laboratório de Psicologia, Instituto Superior de Psicologia Aplicada, Portugal, v.4, n. 1, p.65-90.

Moesby, E. (2008) What is an effective approach to introducing $P B L / P O P B L$ in an institution: a model for making the change to $P B L / P O P B L$ as an alternative approach to Higher Education Institutions. $2^{\mathrm{a}}$. Edição. Aalborg: Institut for Development and Planning, Aalborg Universitet.

Pestana, M. H e J. N. Gageiro (2003) Análise de dados para ciências sociais: a complementaridade do SPSS. 3 edição, Lisboa, Ed. Silabo.

Ruben, B. (1999) Simulations, Games, and Experience-Based Learning: The Quest for a New Paradigm for Teaching and Learning. Simulation \& Gaming, v. 30 n. 4, p. 498-505. DOI: 10.1177/ 104687819903000409 .

Savery, J. R. (2006) Overview of Problem-based Learning: Definitions and Distinctions. The Interdisciplinary Journal of Problem-based Learning, v. 1, n. 1, p. 9-20. DOI: 10.7771/1541-5015.1002.

Silva, A. N. R.; N. P. Kuri e A. Casale (2012) PBL and B-Learning for Civil Engineering Students in a Transportation Course. Journal of Professional Issues in Engineering, Education and Practice (no prelo). DOI: 10.1061/(ASCE)EI.1943-5541.0000115.

Yeo, R. (2005) Problem-based learning: lessons for administrators, educators and learners. International Journal of Educational Management, v. 19 n. 7, p. 541-551. DOI 10.1108/09513540510625581. 